\title{
Subcortical connectivity in dementia with Lewy bodies and Alzheimer's disease
}

\author{
Eva R. Kenny, John T. O'Brien, Michael J. Firbank and Andrew M. Blamire
}

\section{Background}

Resting-state functional magnetic resonance imaging (fMRI) can be used to measure correlations in spontaneous lowfrequency fluctuations in the blood oxygen level-dependent (BOLD) signal which represent functional connectivity between key brain areas.

\section{Aims}

To investigate functional connectivity with regions hypothesised to be differentially affected in dementia with Lewy bodies (DLB) compared with Alzheimer's disease and controls.

\section{Method}

Fifteen participants with probable DLB, 16 with probable Alzheimer's disease and 16 controls were scanned in the resting-state using a $3 \mathrm{~T}$ scanner. The BOLD signal time-series of fluctuations in seed regions were correlated with all other voxels to measure functional connectivity.

\section{Results}

Participants with DLB and Alzheimer's disease showed greater caudate and thalamic connectivity compared with controls. Those with DLB showed greater putamen connectivity compared with those with Alzheimer's disease and the controls. No regions showed less connectivity in DLB or Alzheimer's disease $v$. controls, or in DLB $v$. Alzheimer's disease.

\section{Conclusions}

Altered connectivity in DLB and Alzheimer's disease provides new insights into the neurobiology of these disorders and may aid in earlier diagnosis.

\section{Declaration of interest}

J.T.O'B. has acted as a consultant for GE Healthcare, Servier, and Bayer Schering Pharma; has received speaker honoraria from Pfizer, GE Healthcare, Eisai, Shire, Lundbeck, Eli Lilly \& Company, and Novartis.
Dementia with Lewy bodies (DLB) is the second most common cause of neurodegenerative dementia after Alzheimer's disease. ${ }^{1,2}$ The overlapping clinical symptoms of DLB and Alzheimer's disease make differentiation between the disorders difficult. This has led to the use of neuroimaging methods, for example single photon emission computed tomography (SPECT), positron emission tomography (PET), and structural and functional magnetic resonance imaging (MRI), to enhance diagnostic accuracy. Neuroimaging has provided important information on differences in specific structures between individuals with Alzheimer's disease and DLB that attempt to explain the varying symptoms. ${ }^{3}$ In general, neuroimaging changes have been less well investigated in DLB compared with Alzheimer's disease and the neurobiological changes underpinning the core features in DLB remain unclear.

Using resting-state functional MRI (fMRI), regional correlations in spontaneous low-frequency fluctuations (at $<0.1 \mathrm{~Hz}$ ) in the blood oxygen level-dependent (BOLD) signal have been reported, ${ }^{4}$ and interpreted as depicting functional connectivity. ${ }^{5}$ Resting-state studies in Alzheimer's disease show functional connectivity abnormalities with the hippocampus and posterior cingulate cortex, but results are conflicting, with some reporting decreases, ${ }^{6,7}$ and others increases. ${ }^{8,9}$ Previously, we investigated cortical connectivity in DLB and Alzheimer's disease and using a seed-region approach showed abnormal functional connectivity with the posterior cingulate in DLB and with the hippocampus in Alzheimer's disease. Abnormalities in participants with DLB and Alzheimer's disease were characterised by increased connectivity. ${ }^{10}$ Only one other group has investigated resting-state connectivity in DLB. ${ }^{11}$ Galvin et al focused on precuneus connectivity only and using the whole structure as the seed region showed both increased connectivity with the putamen and inferior parietal cortex and decreased connectivity with the medial prefrontal cortex, frontoparietal operculum and primary visual cortex. $^{11}$
This study used resting-state fMRI to compare participants with DLB with those with Alzheimer's disease and with controls, focusing on subcortical connectivity from the caudate nucleus, putamen and thalamus. Connectivity was hypothesised to be abnormal in individuals with DLB compared with those with Alzheimer's disease and the controls in the following regions: the caudate, because of its role in emotional regulation and the greater depression severity in DLB; the putamen, because abnormalities in structural pathology and neurotransmitter function here are associated with Parkinsonian symptoms in DLB; ${ }^{12,13}$ and the thalamus (mediodorsal nucleus), because it is involved in the maintenance of consciousness ${ }^{14}$ and fluctuating cognition is a core feature of DLB. ${ }^{1}$

\section{Method}

\section{Participants}

The study involved 47 participants aged over 60 years: 15 with DLB, 16 with Alzheimer's disease and 16 controls. Recruitment of the participants with DLB and Alzheimer's disease was from clinical old age psychiatry, geriatric medicine and neurology out-patient services and the controls by local advertisement or from partners of participants. The participants were the same as those in a previous paper. ${ }^{15}$ The study was approved by the local ethics committee and all participants gave signed informed consent for participation, following an explanation of the full procedure by an experienced clinician. The participants with DLB (DLB group) met consensus criteria for probable DLB: presence of two or more core features (fluctuating cognition, visual hallucinations and/or Parkinsonism $)^{1,16}$ and the participants with Alzheimer's disease (Alzheimer's disease group) fulfilled National Institute of Neurological and Communicative Diseases and Stroke/Alzheimer's Disease and Related Disorders Association (NINCDS/ADRDA) criteria for probable Alzheimer's disease. ${ }^{17}$ 
Diagnoses were made by consensus between two experienced clinicians, a method previously validated against autopsy diagnosis. ${ }^{18}$ Of the 15 individuals in the DLB group, 9 had undergone a ${ }^{123}$ I-labelled N-(3-fluoropropyl)-2 $\beta$-carbomethoxy$3 \beta$-(4-iodophenyl) nortropane $\left({ }^{123}\right.$ I-FP-CIT) SPECT scan as part of their clinical diagnostic assessment and all demonstrated reduced dopamine transporter uptake in the basal ganglia consistent with their diagnosis.

Detailed physical, neurological and neuropsychiatric examinations were carried out. Cognitive and neuropsychiatric examinations involved: Mini-Mental State Examination $(\mathrm{MMSE})^{19}$ and Cambridge Cognitive (CAMCOG) examination to assess cognitive status, ${ }^{20}$ Geriatric Depression Scale (GDS) to assess depressive symptoms, ${ }^{21}$ Neuropsychiatric Inventory (NPI) to assess neuropsychiatric symptoms, ${ }^{22}$ Clinical Assessment of Fluctuation Scale (CAFS) to assess fluctuating cognition ${ }^{23}$ and the motor subsection of the Unified Parkinson's Disease Rating Scale (UPDRS III) for motor features of Parkinsonism. ${ }^{24}$ Exclusion criteria were severe concurrent illness (apart from dementia in the DLB and Alzheimer's disease groups), the presence of space occupying lesions on MRI, stroke history and any contraindications to MRI. Controls had no history of psychiatric illnesses as self-reported and as assessed by an experienced psychiatrist.

\section{Imaging}

Participants were scanned using a 3 Tesla MRI system (Intera Achieva scanner, Philips Medical System, Eindhoven, The Netherlands). An 8-channel head coil was used to collect resting-state fMRI scans using a gradient-echo echo-planar imaging sequence. The scan timings and parameters were: 25 axial slices, 128 volumes, anterior-posterior acquisition, in-plane resolution: $2 \times 2 \mathrm{~mm}$, slice thickness: $6 \mathrm{~mm}$, repetition time $(\mathrm{TR})=3000 \mathrm{~ms}$, echo time $(\mathrm{TE})=40 \mathrm{~ms}$, field of view: $260 \times 150 \times 260 \mathrm{~mm}$, acquisition time: $6.65 \mathrm{~min}$. Conventional structural threedimensional $T_{1}$-weighted scans were also collected.

\section{Image analysis}

Analysis used the methods described by Fox et al: ${ }^{25}$ removal of non-brain structures, head motion correction, spatial smoothing (6 $\mathrm{mm}$ full-width at half maximum) and temporal band-pass filtering between 0.009 and $0.08 \mathrm{~Hz}$ to remove low-frequency drift and high-frequency noise respectively. A study-specific functional brain template was created as the participants in this study were elderly, therefore they would be expected to have more brain atrophy than that in a general standard space template, which is based on younger individuals. To create the study-specific brain template, one participant was registered to the standard space echo planar imaging template from Statistical Parametric Mapping (SPM5, www.fil.ion.ucl.ac.uk/spm/), all other participants were registered to this individuals and then averaged using fslmaths. Seeds that had been placed in patient space were transformed to standard space for analysis. All analysis was performed using standard tools from the FSL software package (version 5, http://fsl.fmrib.ox.ac.uk/fsl/fslwiki/). ${ }^{26}$

Seed regions of $2 \times 2$ voxels $(446 \mathrm{~mm}$ ) were placed manually for each participant on the functional image in the left and right head of caudate nucleus, putamen and thalamus (mediodorsal nucleus). The mean BOLD signal time-series was extracted from each seed and cross-correlated with all other brain voxels to determine functional connectivity. ${ }^{27}$ To ensure non-neuronal fluctuations did not confound analysis, time series from seeds placed in white matter and cerebrospinal fluid, and a whole brain mask to remove the global signal, were included in the linear regression analysis as covariates of no interest. ${ }^{25}$

A three-group comparison was carried out to investigate connectivity differences between groups for each seed region, by comparing their data on a voxel by voxel basis. ${ }^{28,29} Z$ (Gaussianised $T / F)$ statistic images were thresholded using pixel clusters determined by $Z>2.3 / P<0.05$ (corrected for multiple comparisons). ${ }^{30}$ The peak connectivity cluster coordinates were converted from Montreal Neurological Index (MNI) space to Talairach space ${ }^{31}$ using GingerALE (version 2.1, www.brainmap.org) on Windows ${ }^{32}$ and entered into Talairach Client to assign Talairach labels. ${ }^{33}$ Oneway analysis of variance was used to compare demographic factors across groups and the independent-sample $t$-test for comparisons between groups $(P \leqslant 0.05)$ using SPSS (version 15.0.1) on Windows.

\section{Results}

\section{Demographics}

Table 1 shows the clinical characteristics of the study participants. Groups were comparable for age $(P=0.29$, d.f. $=2, F=1.26)$ and gender $\left(P=0.47, \chi^{2}=0.532\right.$, d.f. $\left.=1\right)$. As expected, the control group had significantly higher scores on cognitive tests (MMSE and CAMCOG) and lower scores on measures of motor features (UPDRS) and depression (GDS), compared with individuals in the DLB and Alzheimer's disease groups. There were no significant differences between the DLB and Alzheimer's disease groups in age at onset of dementia, duration of dementia, MMSE or total CAMCOG scores. Consistent with the known preservation of memory in DLB, participants in this group had significantly higher scores than those in the Alzheimer's disease group on the CAMCOG memory subscore $(P=0.022)$, although still significantly lower than that of the control group. As expected, UPDRS, NPI, CAFS and GDS scores were significantly higher in the DLB group compared with the Alzheimer's disease group, indicating greater severity in the DLB group of the motor features of Parkinsonism $(P<0.001)$, neuropsychiatric disturbances $(P=0.002)$, fluctuating cognition $(P=0.006)$ and depressive symptoms $(P=0.001)$.

At the time of study, 24 participants were taking acetylcholinesterase inhibitors: 14 participants with Alzheimer's disease (donepezil: $n=9$ and galantamine: $n=5$ ) and 10 with DLB (donepezil: $n=5$, galantamine: $n=4$ and rivastigmine $n=1$ ). No participants were on memantine. Eight participants (six with DLB and two with Alzheimer's disease) were taking antidepressants (citalopram, mirtazapine, trazodone, venlafaxine or paroxetine) and one person with DLB was taking a non-benzodiazepine (zopiclone) as a hypnotic.

\section{Caudate functional connectivity}

Online Fig. DS1 shows the connectivity maps for the left and right caudate in each group (the coordinates are provided in online Table DS1). Statistical comparison of group results showed significant differences in connectivity between the DLB and control groups bilaterally with the head of caudate nucleus (Fig. DS1) and between the Alzheimer's disease and control groups for the right caudate (Fig. DS1(b)). In the DLB compared with the control group the left caudate showed abnormal connectivity with the parahippocampal gyrus (left), posterior cingulate (right) and precuneus (bilateral). The right caudate showed abnormal connectivity in the DLB compared with the control group with the posterior cingulate (bilateral), precuneus (left) and culmen (right), and in the Alzheimer's disease compared with the control group with the posterior cingulate (bilateral) and precuneus/ cuneus (bilateral) (Fig. DS1 and Table DS1). In the DLB group, 


\begin{tabular}{|c|c|c|c|c|}
\hline Demographic and neuropsychological data & $\begin{array}{l}\text { Dementia with Lewy } \\
\text { bodies group }(n=15)\end{array}$ & $\begin{array}{l}\text { Alzheimer's disease } \\
\text { group }(n=16)\end{array}$ & $\begin{array}{l}\text { Control group } \\
\qquad(n=16)\end{array}$ & $P$ \\
\hline Age, years: mean (s.d.) & $80.6(6.0)$ & $77.3(8.9)$ & $76.3(8.3)$ & $0.294^{a}$ \\
\hline Gender, male:female: $n$ & $9: 6$ & $8: 8$ & $9: 7$ & $0.466^{\mathrm{b}}$ \\
\hline Age at onset of dementia, years: mean (s.d.) & $77.2(6.7)$ & $73.9(8.9)$ & & $0.256^{\mathrm{c}}$ \\
\hline Duration of dementia, months: mean (s.d.) & $40.2(20.3)$ & $40.4(24.8)$ & & $0.981^{\mathrm{C}}$ \\
\hline \multicolumn{5}{|l|}{ Tests, mean (s.d.) } \\
\hline Mini-Mental State Examination ${ }^{d}$ & $19.5(4.2)$ & $21.1(3.5)$ & $28.6(1.3)$ & $<0.001^{\mathrm{a}}$ \\
\hline Cambridge Cognitive Examination, total ${ }^{\mathrm{e}}$ & $69.0(12.9)$ & $68.9(11.4)$ & $96.9(3.5)$ & $<0.001^{\mathrm{a}}$ \\
\hline Cambridge Cognitive Examination, memory ${ }^{f}$ & $16.0(4.9)$ & $12.1(4.0)$ & $24.1(2.1)$ & $<0.001^{\mathrm{a}}$ \\
\hline Unified Parkinson's Disease Rating Scale, subsection $\|^{g}$ & $22.1(11.9)$ & $6.1(4.4)$ & $2.7(3.6)$ & $<0.001^{a}$ \\
\hline Neuropsychiatric Inventory ${ }^{\text {h }}$ & $23.1(11.5)$ & $8.5(11.8)$ & & $0.002^{\mathrm{C}}$ \\
\hline Clinical Assessment of Fluctuation Scale ${ }^{i}$ & $6.7(5.3)$ & $1.6(3.4)$ & & $0.01^{\mathrm{c}}$ \\
\hline Geriatric Depression Score & $7.1(3.3)$ & $3.4(2.4)$ & $1.3(1.4)$ & $<0.001^{\mathrm{a}}$ \\
\hline \multicolumn{5}{|c|}{ 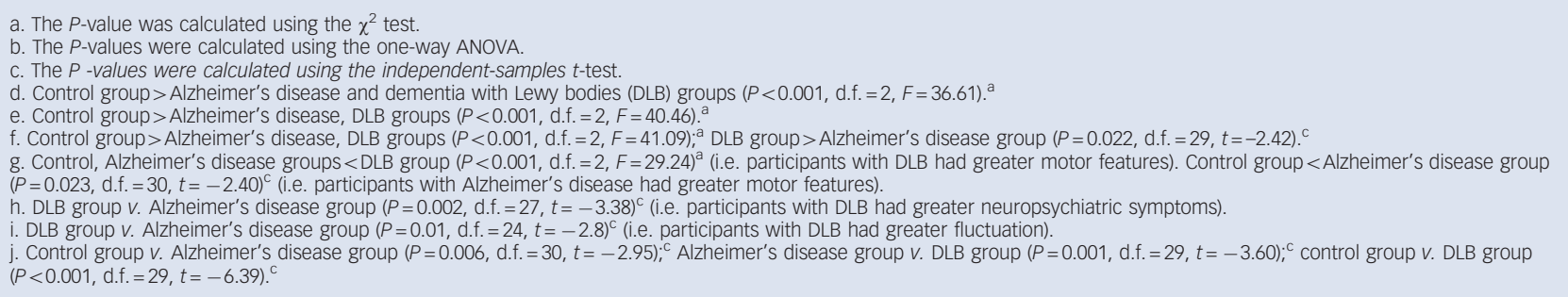 } \\
\hline
\end{tabular}

there were no regions of significantly less connectivity compared with the Alzheimer's disease or control groups, or in the Alzheimer's disease group compared with the control group.

\section{Putamen functional connectivity}

Selected putamen connectivity maps for each group are shown in online Fig. DS2 (the coordinates are provided in online Table DS2). Altered putamen connectivity was specific to the DLB group. Group comparisons showed abnormal connectivity in the DLB compared with the Alzheimer's disease group between the putamen (bilateral) and pre- and postcentral gyrus, inferior parietal and transverse temporal regions (all left hemisphere), and between the DLB and control groups with the left putamen and pre- and postcentral gyrus and inferior parietal regions (all left hemisphere) (Fig. DS2 and Table DS2).

\section{Thalamus functional connectivity}

Thalamic connectivity for each group is shown in online Fig. DS3 (the coordinates are provided in online Table DS3). Aberrant connectivity was found in the DLB and Alzheimer's disease groups compared with controls with the left thalamus (Fig. DS3(a)), and in the DLB group compared with the control group with the right thalamus (Fig. DS3(b)). Abnormal left thalamus connectivity was shown in the DLB group compared with the controls with the cingulate (bilateral), insula (right) and frontal regions (bilateral) and in the Alzheimer's disease group compared with controls with the pre- and postcentral gyrus and inferior parietal regions (all right hemisphere). For the right thalamus, the DLB group showed altered connectivity compared with the control group in frontal and limbic regions (all right hemisphere) (Fig. DS3 and Table DS3). The DLB and Alzheimer's disease groups did not show less connectivity than controls between the thalamus (bilateral) and any other brain regions.

\section{Discussion}

Resting-state fMRI was used to investigate functional connectivity in participants with DLB compared with participants with
Alzheimer's disease and controls. The main findings were abnormal connectivity between subcortical and cortical regions in the DLB group, and to a lesser extent the Alzheimer's disease group, compared with controls. In the DLB group, compared with controls, greater connectivity was seen with the head of caudate nucleus (bilateral), putamen (left) and mediodorsal nucleus of the thalamus (bilateral). In the Alzheimer's disease group compared with controls connectivity was greater with the caudate nucleus (right) and thalamus (left), and in the DLB group compared with the Alzheimer's disease group with the putamen (bilateral). The DLB and Alzheimer's disease groups did not show significantly less connectivity with any seeds compared with controls, the DLB group did not show less connectivity compared with the Alzheimer's disease group.

Compared with controls, the DLB and Alzheimer's disease groups showed altered connectivity in some common brain regions; between the caudate and limbic (posterior cingulate cortex) and parietal (precuneus) regions and between the thalamus and frontal (precentral gyrus) regions. However, there were also important differences between the DLB and Alzheimer's disease groups even though the groups were matched for age and dementia severity. The DLB group showed altered connectivity between the putamen and frontal (left precentral gyrus), temporal (left transverse temporal gyrus) and parietal (left inferior parietal and postcentral gyrus) regions compared with the Alzheimer's disease group.

\section{Caudate}

Previous studies using SPECT and diffusion tensor imaging (DTI) have shown abnormalities in the caudate nucleus in individuals with $\mathrm{DLB}^{12,34}$ and using resting-state fMRI we showed greater caudate functional connectivity in people with late-life depression compared with controls. ${ }^{35}$ In this study, participants with DLB showed greater connectivity compared with controls between the caudate (bilaterally) and default mode network (a resting-state network involved in attending to environmental stimuli) ${ }^{36}$ regions of the posterior cingulate cortex and precuneus (bilaterally). In the 
Alzheimer's disease group, connectivity was greater compared with controls between the caudate (right) and the posterior cingulate cortex and precuneus (bilaterally). The findings here of greater caudate connectivity (bilaterally) in those with DLB could be related to the role the caudate plays in emotional regulation. Depression is known to be more common in people with $\mathrm{DLB}^{16}$ and consistent with this the DLB group in this study showed significantly greater depression severity than those with Alzheimer's disease and the controls.

\section{Putamen}

Neuroimaging studies investigating the putamen in people with DLB have previously shown greater atrophy on structural $\mathrm{MRI},{ }^{37}$ tissue organisation abnormalities on $\mathrm{DTI}^{34}$ and dopamine transporter loss on SPECT. ${ }^{12}$ Importantly, Cousins et $a l^{37}$ and O'Brien et al $^{12}$ showed that these abnormalities are not present in Alzheimer's disease and so are specific to DLB. In this study we also showed that the putamen is affected in DLB, with the DLB group showing greater connectivity with the putamen compared with the Alzheimer's disease and control groups. Connectivity was greater in the DLB group compared with the Alzheimer's disease (bilateral) and control (left) groups in similar brain regions: frontal (precentral), parietal (inferior and postcentral) and temporal (transverse) (all left hemisphere). Altered putamen connectivity was specific to DLB, with no other significant differences between groups found. The putamen is involved in the control of motor functions, therefore abnormalities in connectivity with this structure in DLB could be associated with Parkinsonian symptoms that are characteristic of DLB.

\section{Thalamus}

The thalamus is thought to be involved in maintenance of consciousness $^{14}$ and at post-mortem increased nicotinic receptor binding is shown in individuals with DLB with disturbances of consciousness compared with individuals with DLB but without such disturbances and controls. ${ }^{38}$ The DLB group showed altered connectivity compared with controls between the thalamus (bilaterally) and frontal (precentral gyrus) and limbic (cingulate) (mainly right hemisphere) regions. The Alzheimer's disease group showed altered connectivity compared with controls between the left thalamus and frontal (precentral gyrus) and parietal (inferior parietal and postcentral gyrus) regions (all right hemisphere). The thalamus had not been predicted to be affected in Alzheimer's disease, but previously structural measures have shown greater thalamic grey matter loss in Alzheimer's disease than DLB. ${ }^{39}$ Similar to the findings for the caudate, connectivity was affected bilaterally in the DLB group and unilaterally in the Alzheimer's disease group. Changes in connectivity between the thalamus and cingulate, both key areas implicated in attention, were specific to the DLB group.

\section{Findings in relation to other studies}

Resting-state functional connectivity in Alzheimer's disease has previously been investigated by a number of groups, but studies have generally focused on using posterior cingulate cortex or hippocampus seeds only and findings have differed with some reporting increased, ${ }^{8,9}$ others decreased ${ }^{6,7}$ and within the same study both increased and decreased ${ }^{40,41}$ connectivity in Alzheimer's disease. Similar to our findings in this present study, we previously showed, using cortical seed regions, greater/ increased connectivity in individuals with DLB and Alzheimer's disease compared with controls. Connectivity with a seed placed in the posterior cingulate cortex (right) was increased in participants with DLB compared with controls, whereas hippocampal (left) connectivity was increased in participants with Alzheimer's disease compared with controls. ${ }^{10}$

There has only been one study outside our group investigating resting-state connectivity in DLB and this study used a different approach investigating bilateral precuneus connectivity only and reported both increased (with the putamen and inferior parietal cortex) and decreased (medial prefrontal cortex, frontoparietal operculum and primary visual cortex) connectivity. ${ }^{11}$ However, that study ${ }^{11}$ was in younger participants with DLB (mean age 72 years) with a higher MMSE score (mean 25) compared with our group (mean age 81 years, mean MMSE 20). As the participants in the Galvin et al study ${ }^{11}$ are at an earlier stage of dementia, this could mean that they are better able to compensate for brain damage by increasing connectivity with some brain regions and decreasing connectivity with others.

A task-state study in participants with DLB showed significantly decreased deactivations (for example posterior cingulate cortex) in individuals with DLB and Alzheimer's disease compared with controls. ${ }^{42}$ This could be linked with our finding of increased connectivity between the caudate and posterior cingulate in the DLB and Alzheimer's disease groups compared with controls. This proposes the theory that mechanisms to suppress a region when it is not required in the task state may be inhibited in dementia and therefore regions not required for normal brain functioning at rest remain abnormally active. It could also be that there is a dysfunction in the competitive/opposing relationship between brain regions known to deactivate when a task is performed (default mode network regions) and brain regions known to activate when a task is performed (task positive/attentional networks). ${ }^{25}$ The findings from this study support the compensatory recruitment hypothesis. ${ }^{9}$ Regions not affected in dementia could be recruited to compensate for the poorer functioning of affected regions, therefore greater connectivity is observed as circuits compensate for damage elsewhere.

\section{Strengths and limitations}

Strengths of this study were the good match of groups for participant numbers, gender and age. The model-based technique used requires prior hypotheses for seed selection, which were formed based on previous neuroimaging study findings showing these regions to be abnormal, and enabled the investigation of spontaneous low-frequency fluctuation correlations between a seed and all other voxels.

This resting-state fMRI study investigated connectivity in DLB with subcortical brain regions. Previous studies in Alzheimer's disease have used different seeds and have either investigated connectivity between two specific seeds or within a seed only, thus potentially missing important changes elsewhere in the brain. A previous study in DLB placed a different seed (precuneus) bilaterally using coordinates. ${ }^{11}$ In contrast, we placed seeds manually in each participant, meaning the seed was less likely to be affected by atrophy differences between participants, and connectivity was investigated separately for each hemisphere. Resting-state studies benefit from simplicity of experimental design, no task has to be practised or performed, meaning they are advantageous in individuals who are cognitively impaired for whom it may be more difficult to adhere to a task.

This study also has some limitations. The results may be biased by the seed selected and connectivities of interest may be missed if they do not show connectivity with the seed. In contrast, model-free methods do not require predefined seeds or a temporal model, although their lack of specificity means results can be hard to interpret. The number of participants in each group was 
relatively small; with larger group sizes more significant differences may have been observed. However, other resting-state fMRI studies have had comparable numbers, ${ }^{9}$ and others have had less. ${ }^{6}$ Additionally, participants can have overlapping DLB and Alzheimer's disease pathology and therefore subsequent autopsy correlation with pathological burden, as has been carried out in structural MRI studies, ${ }^{43}$ would be informative.

The participants in the DLB and Alzheimer's disease groups in this study were not medication free and as certain medications have been shown to affect functional connectivity this must be taken into account. The majority of the participants in the DLB and Alzheimer's disease groups (24 out of 31) were taking acetylcholinesterase inhibitors at the time of scanning, with all participants (apart from one) taking either donepezil or galantamine. Previously, medications have been shown to decrease connectivity, for example an electroencephalogram (EEG)/fMRI study showed donepezil decreased default mode network connectivity in healthy older participants ${ }^{44}$ and a study investigating antidepressant treatment (citalopram) showed decreased functional connectivity in control participants between the amygdala and ventral medial prefrontal cortex. ${ }^{45}$ These findings provide support for our study findings not being related to medication effects as we showed increased connectivity in the DLB and Alzheimer's disease groups compared with controls. Additionally, we identified significantly different putamen connectivity patterns in the DLB compared with the Alzheimer's disease group, despite the participants taking similar medications and even though more participants with Alzheimer's disease (88\%) were taking acetylcholinesterase inhibitors than those with DLB (67\%) we showed greater connectivity in the DLB group.

Memantine, an N-methyl-D-aspartate (NMDA) receptor antagonist, has been shown to increase precuneus resting-state functional connectivity in Alzheimer's disease, ${ }^{46}$ however none of the people with dementia in this study were taking memantine and in a previous study we showed no significant differences in precuneus connectivity in the same participants studied here. ${ }^{10}$ In late-life depression, we reported no significant difference in resting-state caudate functional connectivity between participants taking medication compared with those not, but as group sizes were small this would need to be investigated further. ${ }^{35}$ Increased resting-state functional connectivity has been reported in a number of studies in depression, ${ }^{47,48}$ Sheline et al showed increased connectivity in the affective network (involving the caudate) in depression. ${ }^{48}$ The DLB group in this study had significantly greater depression severity (as assessed by GDS score) compared with the Alzheimer's disease and control groups, and similarly to Sheline et al we show increased connectivity with the caudate in the DLB group, with our connectivity map (Fig. DS1) showing strong similarities with the affective network described by Sheline et al ${ }^{48}$ even though some of our participants were taking antidepressants. It is important that medication effects on connectivity are investigated further, for example before and after treatment and how treatment might affect cognitive measures, however this would comprise a separate study itself.

In conclusion, the main findings of this study were in individuals with DLB greater subcortical functional connectivity with the head of caudate nucleus, which may be related to depressive symptoms; the putamen, which may be as a result of motor symptoms of Parkinson's disease; and the thalamus, which may be linked to fluctuations in cognition. These results are in agreement with our previous study that also showed greater connectivity in DLB and Alzheimer's disease with cortical seed regions. ${ }^{10}$ In combination, these studies provide support for the theory that in DLB and Alzheimer's disease connectivity may increase in the earlier stages of disease with new brain areas recruited to compensate for loss of others, before activity is decreased later in the course of the disease. Further studies investigating longitudinal changes and treatment effects are needed to explore these hypotheses further.

\section{Funding}

This work was supported by the Medical Research Council, Alzheimer's Research UK and the UK NIHR Biomedical Research Centre for Ageing and Age-related Disease award to the Newcastle upon Tyne Hospitals NHS Foundation Trust.

\section{Acknowledgements}

The authors thank the study participants for their invaluable contribution and the North East Dementia and Neurodegenerative Diseases Research Network (DeNDRoN) for help with participant recruitment.

Eva R. Kenny, PhD, Newcastle Magnetic Resonance Centre and Institute for Ageing and Health, Newcastle University, Wolfson Research Centre, Campus for Ageing and Vitality, Newcastle upon Tyne; John T. O'Brien, DM, FRCPsych, Michael J. Firbank, PhD, Institute for Ageing and Health, Newcastle University, Wolfson Research Centre, Campus for Ageing and Vitality, Newcastle upon Tyne; Andrew M. Blamire, PhD, Institute for Cellular Medicine and Newcastle Magnetic Resonance Centre, Campus for Ageing and Vitality, Newcastle University, Newcastle upon Tyne, UK

Correspondence: Eva R. Kenny, Newcastle Magnetic Resonance Centre, Campus for Ageing and Vitality, Newcastle University, Newcastle upon Tyne NE4 5PL, UK. Email: eva.kenny@ncl.ac.uk

First received 5 Jan 2012, final revision 28 Jun 2012, accepted 3 Apr 2013

\section{References}

1 McKeith IG, Galasko D, Kosaka K, Perry EK, Dickson DW, Hansen LA, et al. Consensus guidelines for the clinical and pathologic diagnosis of dementia with Lewy bodies (DLB): report of the consortium on DLB international workshop. Neurology 1996; 47: 1113-24.

2 Geser F, Wenning GK, Poewe W, McKeith I. How to diagnose dementia with Lewy bodies: state of the art. Mov Disord 2005; 20 (suppl 12): S11-20.

3 Small GW. Neuroimaging as a diagnostic tool in dementia with Lewy bodies. Dement Geriatr Cogn Disord 2004; 17 (suppl 1): 25-31.

4 Biswal B, Yetkin FZ, Haughton VM, Hyde JS. Functional connectivity in the motor cortex of resting human brain using echo-planar MRI. Magn Reson Med 1995; 34: 537-41.

5 Fox MD, Raichle ME. Spontaneous fluctuations in brain activity observed with functional magnetic resonance imaging. Nature Rev 2007; 8: 700-11.

6 Gili T, Cercignani M, Serra L, Perri R, Giove F, Maraviglia B, et al. Regional brain atrophy and functional disconnection across Alzheimer's disease evolution. J Neurol Neurosurg Psychiatry 2011; 82: 58-66.

7 Greicius MD, Srivastava G, Reiss AL, Menon V. Default-mode network activity distinguishes Alzheimer's disease from healthy aging: evidence from functional MRI. Proc Natl Acad Sci U S A 2004; 101: 4637-42.

8 Wang $L$, Zang $Y$, He $Y$, Liang $M$, Zhang $X$, Tian $L$, et al. Changes in hippocampal connectivity in the early stages of Alzheimer's disease: evidence from resting state fMRI. Neurolmage 2006; 31: 496-504.

9 Zhang HY, Wang SJ, Xing J, Liu B, Ma ZL, Yang M, et al. Detection of PCC functional connectivity characteristics in resting-state fMRI in mild Alzheimer's disease. Behav Brain Res 2009; 197: 103-8.

10 Kenny ER, Blamire AM, Firbank MJ, O'Brien JT. Functional connectivity in cortical regions in dementia with Lewy bodies and Alzheimer's disease. Brain 2012; 135: 569-81.

11 Galvin JE, Price JL, Yan Z, Morris JC, Sheline YI. Resting bold fMRI differentiates dementia with Lewy bodies vs Alzheimer disease. Neurology 2011; 76: 1797-803.

12 O'Brien JT, Colloby S, Fenwick J, Williams ED, Firbank M, Burn D, et al. Dopamine transporter loss visualized with FP-CIT SPECT in the differential diagnosis of dementia with Lewy bodies. Arch Neurol 2004; 61: 919-25.

13 Walker Z, Costa DC, Walker RW, Shaw K, Gacinovic S, Stevens T, et al. Differentiation of dementia with Lewy bodies from Alzheimer's disease using a dopaminergic presynaptic ligand. J Neurol Neurosurg Psychiatry 2002; 73: 134-40.

14 Perry EK, Perry RH. Neurochemistry of consciousness: cholinergic pathologies in the human brain. Prog Brain Res 2004; 145: 287-99. 
15 Firbank MJ, Blamire AM, Teodorczuk A, Teper E, Burton EJ, Mitra D, et al. High resolution imaging of the medial temporal lobe in Alzheimer's disease and dementia with Lewy bodies. J Alzheimers Dis 2010; 21: 1129-40.

16 McKeith IG, Dickson DW, Lowe J, Emre M, O'Brien JT, Feldman $\mathrm{H}$, et al. Diagnosis and management of dementia with Lewy bodies: third report of the DLB Consortium. Neurology 2005; 65: 1863-72.

17 McKhann G, Drachman D, Folstein M, Katzman R, Price D, Stadlan EM, et al Clinical diagnosis of Alzheimer's disease: report of the NINCDS-ADRDA Work Group under the auspices of Department of Health and Human Services Task Force on Alzheimer's Disease. Neurology 1984; 34: 939-44.

18 McKeith IG, Ballard CG, Perry RH, Ince PG, O'Brien JT, Neill D, et al. Prospective validation of Consensus criteria for the diagnosis of dementia with Lewy bodies. Neurology 2000; 54: 1050-8.

19 Folstein MF, Folstein SE, McHugh PR. "Mini-mental state". A practical method for grading the cognitive state of patients for the clinician. J Psychiatr Res 1975; 12: 189-98.

20 Roth M, Tym E, Mountjoy CQ, Huppert FA, Hendrie H, Verma S, et al. CAMDEX. A standardised instrument for the diagnosis of mental disorde in the elderly with special reference to the early detection of dementia. Br J Psychiatry 1986; 149: 698-709.

21 Sheikh JI, Yesavage JA. Geriatric Depression Scale (GDS). Recent Evidence and Development of a Shorter Version. The Haworth Press, 1986.

22 Cummings JL, Mega M, Gray K, Rosenberg-Thompson S, Carusi DA, Gornbein J. The Neuropsychiatric Inventory: comprehensive assessment of psychopathology in dementia. Neurology 1994; 44: 2308-14.

23 Walker MP, Ballard CG, Ayre GA, Wesnes K, Cummings JL, McKeith IG, et al. The Clinician Assessment of Fluctuation and the One Day Fluctuation Assessment Scale. Two methods to assess fluctuating confusion in dementia. Br J Psychiatry 2000; 177: 252-6.

24 Fahn S, Elton R. Unified Parkinson's disease rating scale. In Recent Developments in Parkinson's Disease (eds S Fahn, CD Marsden, DB Calne, M Goldstein). MacMillan Healthcare Information, 1987.

25 Fox MD, Snyder AZ, Vincent JL, Corbetta M, Van Essen DC, Raichle ME. The human brain is intrinsically organized into dynamic, anticorrelated functional networks. Proc Natl Acad Sci U S A 2005; 102: 9673-8.

26 Smith SM, Jenkinson M, Woolrich MW, Beckmann CF, Behrens TE, Johansen-Berg $\mathrm{H}$, et al. Advances in functional and structural MR image analysis and implementation as FSL. Neurolmage 2004; 23 (suppl 1): S208-19.

27 Woolrich MW, Ripley BD, Brady M, Smith SM. Temporal autocorrelation in univariate linear modeling of FMRI data. Neurolmage 2001; 14: 1370-86.

28 Beckmann CF, Jenkinson M, Smith SM. General multilevel linear modeling for group analysis in FMRI. Neurolmage 2003; 20: 1052-63.

29 Woolrich MW, Behrens TE, Beckmann CF, Jenkinson M, Smith SM. Multilevel linear modelling for FMRI group analysis using Bayesian inference. Neurolmage 2004; 21: 1732-47.

30 Worsley KJ. Statistical analysis of activation images. In Functional MRI: An Introduction to Methods (eds P Jezzard, P Matthews, S Smith): 251-70. Oxford University Press, 2001.

31 Talairach J, Tournoux P. Co-planar Stereotaxic Atlas of the Human Brain: An Approach to Medical Cerebral Imaging. Thieme Medical Publishers, 1988.
32 Lancaster JL, Tordesillas-Gutierrez D, Martinez M, Salinas F, Evans A, Zilles $\mathrm{K}$, et al. Bias between $\mathrm{MNI}$ and Talairach coordinates analyzed using the ICBM-152 brain template. Hum Brain Mapp 2007; 28: 1194-205.

33 Lancaster JL, Woldorff MG, Parsons LM, Liotti M, Freitas CS, Rainey L, et al. Automated Talairach atlas labels for functional brain mapping. Hum Brain Mapp 2000; 10: 120-31.

34 Bozzali M, Falini A, Cercignani M, Baglio F, Farina E, Alberoni M, et al. Brain tissue damage in dementia with Lewy bodies: an in vivo diffusion tensor MRI study. Brain 2005; 128: 1595-604.

35 Kenny ER, O'Brien JT, Cousins DA, Richardson J, Thomas AJ, Firbank MJ, et al. Functional connectivity in late-life depression using resting-state functional magnetic resonance imaging. Am J Geriatr Psychiatry 2010; 18: 643-51.

36 Raichle ME, MacLeod AM, Snyder AZ, Powers WJ, Gusnard DA, Shulman GL. A default mode of brain function. Proc Natl Acad Sci U S A 2001; 98: 676-82.

37 Cousins DA, Burton EJ, Burn D, Gholkar A, McKeith IG, O'Brien JT. Atrophy of the putamen in dementia with Lewy bodies but not Alzheimer's disease: an MRI study. Neurology 2003; 61: 1191-5.

38 Pimlott SL, Piggott M, Ballard C, McKeith I, Perry R, Kometa S, et al. Thalamic nicotinic receptors implicated in disturbed consciousness in dementia with Lewy bodies. Neurobiol Dis 2006; 21: 50-6.

39 Burton EJ, Karas G, Paling SM, Barber R, Williams ED, Ballard CG, et al. Patterns of cerebral atrophy in dementia with Lewy bodies using voxel-based morphometry. Neurolmage 2002; 17: 618-30.

40 Supekar K, Menon V, Rubin D, Musen M, Greicius MD. Network analysis of intrinsic functional brain connectivity in Alzheimer's disease. PLoS Comput Biol 2008; 4: e1000100.

41 Wang $K$, Liang $M$, Wang L, Tian L, Zhang $X$, Li K, et al. Altered functional connectivity in early Alzheimer's disease: a resting-state fMRI study. Hum Brain Mapp 2007; 28: 967-78.

42 Sauer J, Ffytche DH, Ballard C, Brown RG, Howard R. Differences between Alzheimer's disease and dementia with Lewy bodies: an fMRI study of taskrelated brain activity. Brain 2006; 129: 1780-8.

43 Burton EJ, Barber R, Mukaetova-Ladinska EB, Robson J, Perry RH, Jaros E, et al. Medial temporal lobe atrophy on MRI differentiates Alzheimer's disease from dementia with Lewy bodies and vascular cognitive impairment: a prospective study with pathological verification of diagnosis. Brain 2009; 132: 195-203.

44 Balsters JH, O'Connell RG, Martin MP, Galli A, Cassidy SM, Kilcullen SM, et al. Donepezil impairs memory in healthy older subjects: behavioural, EEG and simultaneous EEG/fMRI biomarkers. PLOS One 2011; 6: e24126.

45 McCabe C, Mishor Z. Antidepressant medications reduce subcortical-cortical resting-state functional connectivity in healthy volunteers. Neuroimage 2011; 57: 1317-23.

46 Lorenzi M, Beltramello A, Mercuri N, Canu E, Zoccatelli G, Pizzini FB, et al Effect of memantine on resting state default mode network activity in Alzheimer's disease. Drugs Aging 2011; 28: 205-17.

47 Perrin JS, Merz S, Bennett DM, Currie J, Steele DJ, Reid IC, et al. Electroconvulsive therapy reduces frontal cortical connectivity in severe depressive disorder. Proc Natl Acad Sci U S A 2012; 109: 5464-8.

48 Sheline $\mathrm{YI}$, Price JL, Yan Z, Mintun MA. Resting-state functional MRI in depresssion unmasks increased connectivity between networks via the dorsal nexus. Proc Natl Acad Sci U S A 2010; 107: 11020-5. 